\section{Cellular Physiology

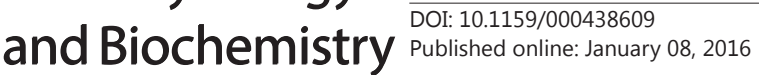

Accepted: September 23, 2015

\title{
Alleviation of Lipopolysaccharides-Induced Acute Lung Injury by MiR-454
}

\author{
Zhengang Tao ${ }^{\mathrm{a}}$ Ying Yuan ${ }^{\mathrm{b}}$ Qingwu Liao \\ aEmergency Department, Zhongshan Hospital, Shanghai, 'bepartment of Geriatrics, Zhongshan \\ Hospital, Shanghai, 'Department of Anesthesiology, Zhongshan Hospital, Shanghai, China
}

\section{Key Words}

Acute lung injury (ALI) • CXCR4 • CXCL12 • MiR-454 • Lipopolysaccharides (LPS)

\begin{abstract}
Background/Aims: Although acute lung injury (ALI) is an important and common disease in humans, its pathogenesis is poorly understood and its therapeutic outcome has not been significantly improved in the past years. Here, we examined whether application of microRNAs might inhibit the ALI-associated lung inflammatory, and subsequently reduce the injury. Methods: In vitro, we performed bioinformatics analyses to identify the miRNAs that target the most important chemo-attractive factor CXCL12, and confirmed that the binding was functional by luciferase reporter assay. We prepared adeno-associated virus (AAV) carrying miRNA mimics or null control. We expressed miRNA in mouse lung through i.v. injection of AAV and then we used Lipopolysaccharides (LPS) to induce ALI in mice. We analyzed the changes in permeability index and production of inflammatory cytokines in mouse lung, and we also verified the effects of virus-mediated gene expression by examining the levels of miRNAs and CXCL12 in lung by RT-qPCR and ELISA, and by quantifying the recruited inflammatory cells in mouse lung by flow cytometry. Results: We found that miR-454 targeted the 3'-UTR of CXCL12 mRNA to inhibit its protein translation in human lung epithelial cells. Overexpression of miR-454 in mouse lung significantly reduced the LPS-induced increases in permeability index and production of inflammatory cytokines CXCL1, CXCL2, IL6 and TNF $\alpha$, possibly through suppression of CXCL12/CXCR4-mediated recruitment of inflammatory cells. Conclusion: Overexpression of miR-454 in lung may be a promising therapeutic approach to reduce the severity of ALI.
\end{abstract}

\section{Introduction}

Acute lung injury (ALI) is an important and common disease in humans, its pathogenesis is poorly understood and its therapeutic outcome has not been significantly improved in the past years [1-4]. The cellular pathology of ALI includes loss of alveolar-capillary 
membrane integrity, excessive trans-epithelial inflammatory cell migration and release of pro-inflammatory cytokines, e.g. interleukin (IL)- 6 and tumor necrosis factor (TNF)- $\alpha, \mathrm{C}-\mathrm{X}-\mathrm{C}$ motif chemokine 1 (CXCL1) and CXCL2. Injuries of lung epithelial and endothelial cells may finally result in loss of respiratory capacity [5-7].

To investigate the molecular mechanisms of ALI in humans, various experimental models of ALI have been used, the most common being the endotoxin model, e.g. Lipopolysaccharides (LPS) [8-11]. In experimental ALI, the lung parenchyma is damaged by the generation and release of proteases and reactive oxygen and nitrogen species produced by inflammatory cells in the interstitial and alveolar compartments, resulting in microvascular injury and diffuse alveolar damage with intrapulmonary hemorrhage, edema, and fibrin deposition [8-11]. Excessive and prolonged inflammation results in basement membrane destruction and increased permeability of the alveolar-capillary barrier. Moreover, inflammatory cells also release pro-inflammatory and pro-apoptotic cytokines to injure adjacent cells to create ulcerating lesions to aggravate the damages of the alveolar-capillary barrier [1-4].

Since inflammatory cells play a pivotal role in the pathogenesis of ALI, it is expected that inflammation suppression may result in reduction of the damage severity of ALI [1-4]. The recruitment, activation and retention of inflammatory cells need crosstalk between chemoattractive factors and ligands. The stromal cell-derived factor 1 (SDF-1) is also known as CXCL12, which is an important member of the chemokine family that activate leukocytes to initiate inflammatory processes [12-14]. CXCL12 is potently chemotactic for inflammatory cells. CXCL12 recruits inflammatory cells through a CXCR4 dependent manner and CXCR4 is expressed in inflammatory cells [12-14]. Recently, CXCL12/CXCR4 axis has been described as a retention signal for M2 macrophages [14, 15]. Hence, suppression of CXCL12/CXCR4 axis may be expected to inhibit inflammation.

MicroRNA (miRNA) is a class of non-coding small RNA that specifically recognizes the $3^{\prime}$-untranslated region (3'-UTR) of the mRNA of some genes through nucleotide pairing [16, 17]. MiRNAs play a critical role in various physiological and pathological events. Among all miRNAs, miR-454 is newly recognized one and has just recently been shown to be induced by TGF $\beta 1$ in hepatic stellate cells and by infection with Schistosoma Japonicum in mouse livers [18], whereas its biological function remains unclear.

Here, we examined whether application of microRNAs might inhibit the ALI-associated lung inflammatory, and subsequently reduce the injury. In vitro, we performed bioinformatics analyses to identify the miRNAs that target CXCL12, and confirmed that the binding was functional by luciferase reporter assay. We prepared adeno-associated virus (AAV) carrying miRNA mimics or null control. We expressed miRNA in mouse lung through i.v. injection of AAV and then we used LPS to induce ALI in these mice. We analyzed the changes in permeability index and virus-mediated gene expression by examining the levels of miRNAs and CXCL12 in lung, and by quantifying the recruited inflammatory cells in mouse lung. We found that miR-454 targeted the 3'-UTR of CXCL12 mRNA to inhibit its protein translation in human lung epithelial cells. Overexpression of miR-454 in mouse lung significantly reduced the LPS-induced increases in permeability index and production of inflammatory cytokines CXCL1, CXCL2, IL6 and TNF $\alpha$, possibly through suppression of CXCL12/CXCR4-mediated recruitment of inflammatory cells.

\section{Materials and Methods}

\section{Animal manipulations}

All mouse experiment protocols were approved by the Animal Research and Care Committee at Zhongshan Hospital. All experiments were performed in accordance with the guidelines from the Animal Research and Care Committee at Zhongshan Hospital. Specific pathogen free (SPF) C57/BL6 mice (female, aged 10 weeks, weight 20 - 24 g) were supplied by Laboratory Animal Center of Shanghai Academy of Sciences, Chinese Academy of Sciences, China. After acclimatization for 1 week, 30 mice receive singled tail vein injection of $10^{9} \mathrm{AAV}$ carrying null construct (AAV-null) ( $\mathrm{n}=20$ ), or $10^{9} \mathrm{AAV}$ carrying miR-454 (AAVmiR-454) ( $n=10)$. One week later, $50 \mu$ g LPS from Escherichia coli (serotype 0111:B4; Sigma-Aldrich, St 


\section{Cellular Physiology Cell Physiol Biochem 2016;38:65-74 \begin{tabular}{l|l|l|}
\hline DOI: 10.1159/000438609 & $\begin{array}{l}\text { C) 2016 The Author(s). Published by S. Karger AG, Basel } \\
\text { www.karger.com/cpb }\end{array}$
\end{tabular} \\ Tao/Yuan/Liao: Alleviation of LPS-Induced ALI by MiR-454}

Louis, MO, USA) in $40 \mu \mathrm{l}$ PBS was given intratracheally to all AAV-miR-454-injected mice (LPS+AAV-miR-454) and 10 from the 20 mice that had received AAV-null (LPS+AAV-null). The rest 10 mice that had received AAVnull underwent the same procedure with intratracheal injection of saline as control (Saline+AAV-null).

\section{Permeability index}

For retrieval of bronchoalveolar lavage fluids (BALF), airways were flushed with $0.8 \mathrm{ml} \mathrm{PBS}$. If not otherwise noted, the permeability index was determined and BAL fluids were collected $6 \mathrm{~h}$ after lung injury induction. Permeability index as a quantitative marker for vascular leakage. Briefly, BSA was labeled with $125 \mathrm{I}$ by the chloramine $\mathrm{T}$ method. A trace amount of $125 \mathrm{I}-\mathrm{BSA}$ (specific activity $5 \mu \mathrm{Ci} / \mu \mathrm{g}$ ) was added to unlabeled BSA ( $5 \mathrm{mg} / \mathrm{ml}$ in PBS), and $200 \mu \mathrm{l}$ of this solution was injected intravenously. Four hours later, mice were euthanized with i.p. ketamine and blood was collected from the inferior vena cava. The thorax was opened, left atrium incised, and the lung was perfused in situ with PBS via the pulmonary artery. The flushed lungs were removed and permeability index (indicating the extent of pulmonary leakage) was determined by using a gamma counter and expressed as the ratio of counts per min (cpm) in the whole lung versus radioactivity in $100 \mu \mathrm{l}$ of blood.

\section{Cell lines}

Human embryonic kidney 293 cell line (HEK293) [6] and Human lung epithelial cell line HULEC-5a (HULEC) [19] were purchased from ATCC (American Type Culture Collection, Manassas, VA, USA). HULEC$5 \mathrm{a}$ is an immortalized human microvascular endothelial cell Line and was maintained in MCDB131 media (without L-Glutamine, ATCC) supplemented with 10ng/ml Epidermal Growth Factor (EGF, Sigma-Aldrich), $1 \mu \mathrm{g} / \mathrm{ml}$ Hydrocortisone (Sigma-Aldrich), 10 mmol/l Glutamine (Invitrogen, Carlsbad, CA, USA) and 10\% fetal bovine serum (FBS, Invitrogen). Both cells were incubated in a humidified chamber with $5 \% \mathrm{CO}_{2}$ at $37^{\circ} \mathrm{C}$.

\section{AAV preparation and cell transfections with plasmids}

AAV-miR-454 and control AAV-null viruses were prepared according to general protocols. Briefly, we used a pAAV-CMV-GFP plasmid (Clontech, Mountain View, CA, USA), a packaging plasmid carrying the serotype 8 rep and cap genes, and a helper plasmid carrying the adenovirus helper functions (Applied Viromics, LLC. Fremont, CA, USA) in this study. The transgenes as-miR-454 or null were inserted between CMV promoter and GFP reporter. $2 \mathrm{~A}$ sequence was used to connect transgene and GFP to allow co-expression of both under the control of the CMV promoter. The transgenes were subcloned into the plasmid using the 50-EcoRI and 30-NheI sites of the pAAV-CMV-GFP vector. Sequencing was performed to confirm the correct orientation of the generated plasmids. AAVs were prepared by triple transfection of the newly prepared plasmids, R2C8 (containing AAV2 Rep and AAV8 capsid genes) and plAd5 (containing adenovirus helper genes) into HEK293 cells by Lipofectamine 2000 reagent (Invitrogen). The viruses were purified using CsCl density centrifugation and then titered by a quantitative densitometric dot-blot assay.

For cell transfection in vitro, the HULEC cells were incubated with plasmids (miR-454 and null) at a MOI of 100 for 12 hours.

RNA extraction, reverse transcription and quantitative RT-PCR (RT-qPCR)

Total RNA and miRNAs were extracted from lung or from the cultured cells with miRNeasy mini kit or RNeasy kit (Qiagen, Hilden, Germany), respectively. Complementary DNA (cDNA) was randomly primed from $2 \mu \mathrm{g}$ of total RNA using the Omniscript reverse transcription kit (Qiagen). RT-qPCR was subsequently performed in triplicate with a 1:4 dilution of cDNA using the Quantitect SyBr green PCR system (Qiagen) on a Rotorgene 6000 series PCR machine. Quantitative PCR (RT-qPCR) were performed in duplicates with QuantiTect SYBR Green PCR Kit (Qiagen). All primers were purchased from Qiagen. Data were collected and analyzed with the Rotorgene software accompanying the PCR machine, using $2^{-\Delta \Delta C t}$ method for quantification of the relative mRNA expression levels. Values of genes were first normalized against $\alpha$-tubulin, and then compared to controls.

ELISA

ELISA for mouse IL6, TNF $\alpha$, CXCL1, CXCL2, CXCL12 used ELISA kits (R\&D Systems, Los Angeles, CA, USA), according to the manufacturer's instruction. 


\section{Cellular Physiology Cell Physiol Biochem 2016;38:65-74 and Biochemistry Published online: January 08, $2016 \quad \begin{aligned} & \text { DOI: 10.1159/000438609 } 2016 \text { The Author(s). Published by S. Karger AG, Basel } \\ & \text { www.karger.com/cpb }\end{aligned}$ \\ Tao/Yuan/Liao: Alleviation of LPS-Induced ALI by MiR-454}

Bioinformatics analyses and luciferase-reporter assay

Targeting miRNAs for 3'-UTR of CXCL12 mRNA were predicted using TargetScan (https://www. targetscan.org) [20]. Luciferase-reporters were successfully constructed using molecular cloning technology. Target sequence for CXCL12 miRNA 3'-UTR clone was purchased from Creative Biogene (Shirley, NY, USA). HULEC-miR-454 or HULEC-null was seeded in 24-well plates for 24 hours, after which they were transfected with $1 \mu \mathrm{g}$ of Luciferase-reporter plasmids per well using PEI Transfection Reagent. Luciferase activities were measured using the dual-luciferase reporter gene assay kit (Promega, Beijing, China), according to the manufacturer's instructions.

Statistical analysis

All statistical analyses were carried out using the SPSS 18.0 statistical software package. All values are depicted as mean \pm standard deviation from 5 individuals and are considered significant if $\mathrm{p}<0.05$. All data were statistically analyzed using one-way ANOVA with a Bonferroni correction, followed by a Fisher's exact test, as necessary.

\section{Results}

MiR-454 targets the 3'-UTR of CXCL12 mRNA to inhibit its protein translation in human lung epithelial cells

In vitro, we performed bioinformatics analyses to identify the miRNAs that target the most important chemo-attractive factor CXCL12, and used luciferase reporter assay to confirm that the binding was functional. Specifically, we found that miR-454 binding sites on the $3^{\prime}$-UTR of CXCL12 mRNA ranged from $1466^{\text {th }}$ to $1472^{\text {nd }}$ base site (Fig. 1A). In order to prove that this binding is functional, we modified miR-454 levels in HULEC cells, and got HULEC-miR-454, HULEC-null (as a control) and HULEC-as-miR-454 cells. The plasmid constructs also contained a GFP reporter to allow examination of transfection efficiency and to allow purification of the transfected cells (Fig. 1B). Next, the levels of miR-454 in these cells were confirmed by RT-qPCR (Fig. 1C). These miR-454-modified cells were then transfected with $1 \mu \mathrm{g}$ of CXCL12-3'-UTR luciferase-reporter plasmid. The luciferase activities in these cells showed that miR-454 targeted 3-'UTR of CXCL12 mRNA to inhibit its translation (Fig. 1D). Moreover, modification of miR-454 levels in HULEC cells did not alter mRNA of CXCL12 (Fig. 1E), but significantly altered the CXCL12 protein (Fig. 1F).

Overexpression of miR-454 in lung alleviates LPS-induced ALI

We then used these plasmids to prepare adeno-associated virus (AAV) carrying miR454 (AAV-miR-454), or null (AAV-null). Then, 30 female C57BL/C mice received single tail vein injection of $10^{9} \mathrm{AAV}$-null $(\mathrm{n}=20)$, or $10^{9} \mathrm{AAV}$-miR-454 $(\mathrm{n}=10)$. One week later, $50 \mu \mathrm{g}$ LPS from Escherichia coli in $40 \mu \mathrm{l}$ PBS was given intratracheally to all $10 \mathrm{AAV}-\mathrm{miR}$-454-injected mice (LPS+AAV-miR-454) and 10 from the 20 mice that had received AAV-null (LPS+AAVnull). The rest 10 mice that had received AAV-null received intratracheal injection of saline (Saline+AAV-null). We found that overexpression of miR-454 in lung significantly reduced the LPS-induced increases in permeability index 6 hours after LPS administration (Fig. 2A). The histological patterns of lung with LPS-induced ALI were characterized by interstitial and intraalveolar deposits of neutrophils and fibrin, prominence of alveolar macrophages, and intraalveolar hemorrhage, and all these features were attenuated in the mouse lung that overexpressed miR-454 (Fig. 2B).

Production of inflammatory cytokines is reduced in mouse lung that overexpresses miR454

We then examined the levels of inflammatory cytokines in the BALF of these mice, and found that LPS treatment significantly increases the levels of IL6 (Fig. 3A), TNF $\alpha$ (Fig. 3B), CXCL1 (Fig. 3C) and CXCL2 (Fig. 3D). However, these increases in inflammatory cytokines were significantly reduced in AAV-454-treated mice (Fig. 3A-D). 


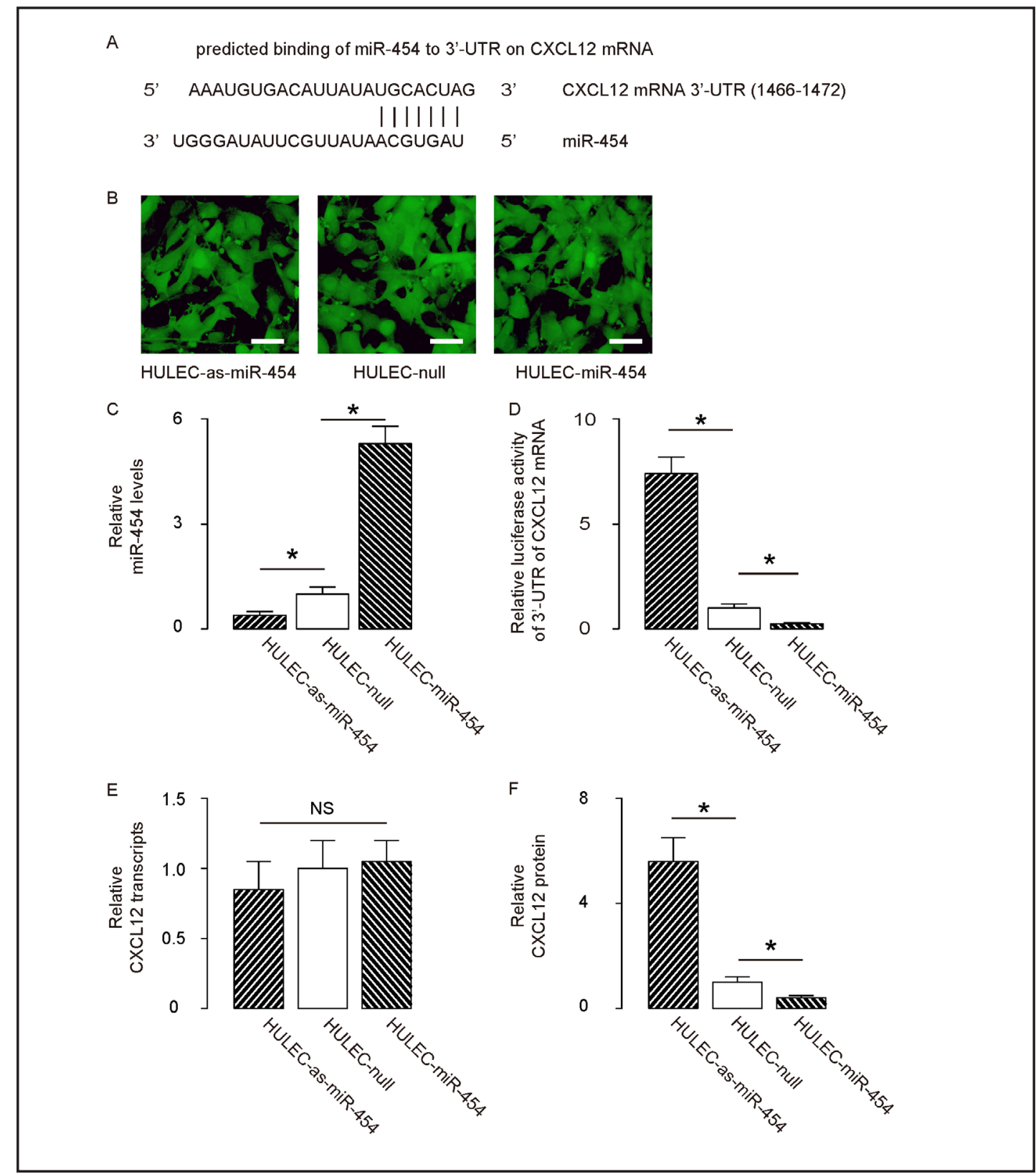

Fig. 1. MiR-454 targets the 3'-UTR of CXCL12 mRNA to inhibit its protein translation in human lung epithelial cells. (A) Bioinformatics analyses showed that miR-454 binding sites on the 3'-UTR of CXCL12 mRNA ranged from $1466^{\text {th }}$ to $1472^{\text {nd }}$ base site. (B) We modified miR-454 levels in HULEC cells, and got HULEC-miR-454, HULEC-null (as a control) and HULEC-as-miR-454 cells. The plasmid constructs also contained a GFP reporter. The transfected cells were in culture. (C) RT-qPCR for miR-454 in these cells. (D) The miR-454-modified cells were then transfected with $1 \mu \mathrm{g}$ of CXCL12-3'-UTR luciferase-reporter plasmid. The luciferase activities in these cells were analyzed. (E-F) The mRNA (E) and protein (F) levels of CXCL12 of miR-454-modified HULEC cells. ${ }^{*} \mathrm{p}<0.05, \mathrm{~N}=5$. Scale bars are $10 \mu \mathrm{m}$.

Overexpression of miR-454 in mouse lung decreases CXCL12 protein but not $m R N A$

We then analyzed the levels of miR-454 by RT-qPCR, the CXCL12 transcripts by RTqPCR, and the CXCL12 protein by ELISA in mouse lung. We verified that AAV-454 injection indeed significantly increased the levels of miR-454 in mouse lung (Fig. 4A). Moreover, AAV454 injection did not alter increases in CXCL12 mRNA in mouse lung from LPS-treated mice (Fig. 4B), but significantly reduced the increases in CXCL12 protein (Fig. 4C). 


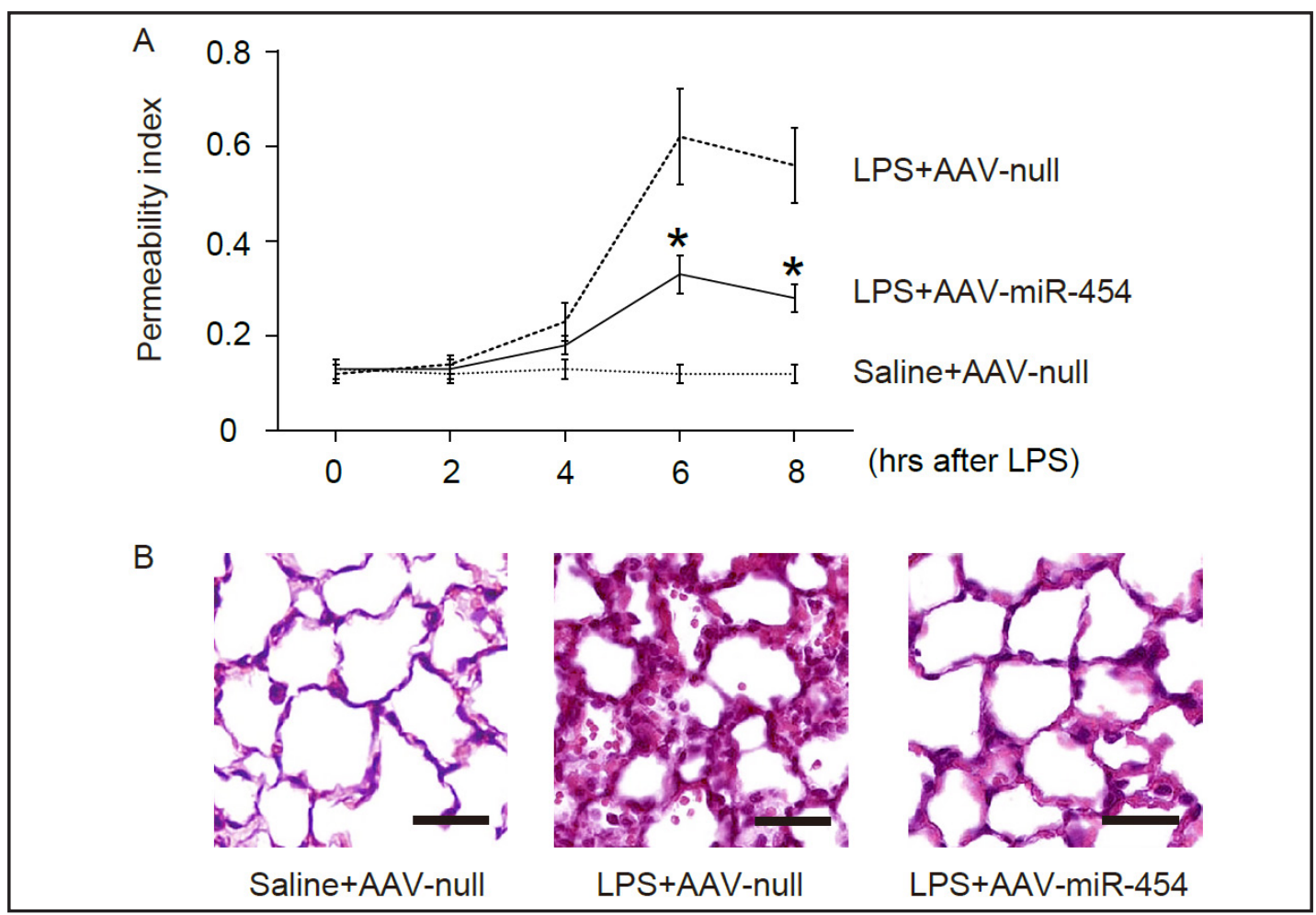

Fig. 2. Overexpression of miR-454 in lung alleviates LPS-induced ALI. Thirty female C57BL/C mice received single tail vein injection of $10^{9} \mathrm{AAV}$-null $(\mathrm{n}=20)$, or $10^{9} \mathrm{AAV}$-miR-454 $(\mathrm{n}=10)$. One week later, 50 $\mu \mathrm{g}$ LPS from Escherichia coli in $40 \mu \mathrm{l}$ PBS was given intratracheally to all $10 \mathrm{AAV}$-miR-454-injected mice (LPS+AAVmiR-454) and 10 from the 20 mice that had received AAV-null (LPS+AAV-null). The rest 10 mice that had received AAV-null received intratracheal injection of saline (Saline+AAV-null). (A) Permeability index. (B) Lung histology. *p < 0.05 (LPS+AAV-miR-454 vs LPS+AAV-null), N = 10. Scale bars are 50 $\mu \mathrm{m}$.

Fig. 3. Production of inflammatory cytokines is reduced in mouse lung that overexpresses miR454. We then examined the levels of inflammatory cytokines in the BALF of these mice by ELISA. (A) IL6 levels, (B) TNFa levels, (C) CXCL1 levels, (D) CXCL2 levels. * $\mathrm{p}<0.05$, $\mathrm{N}=10$.

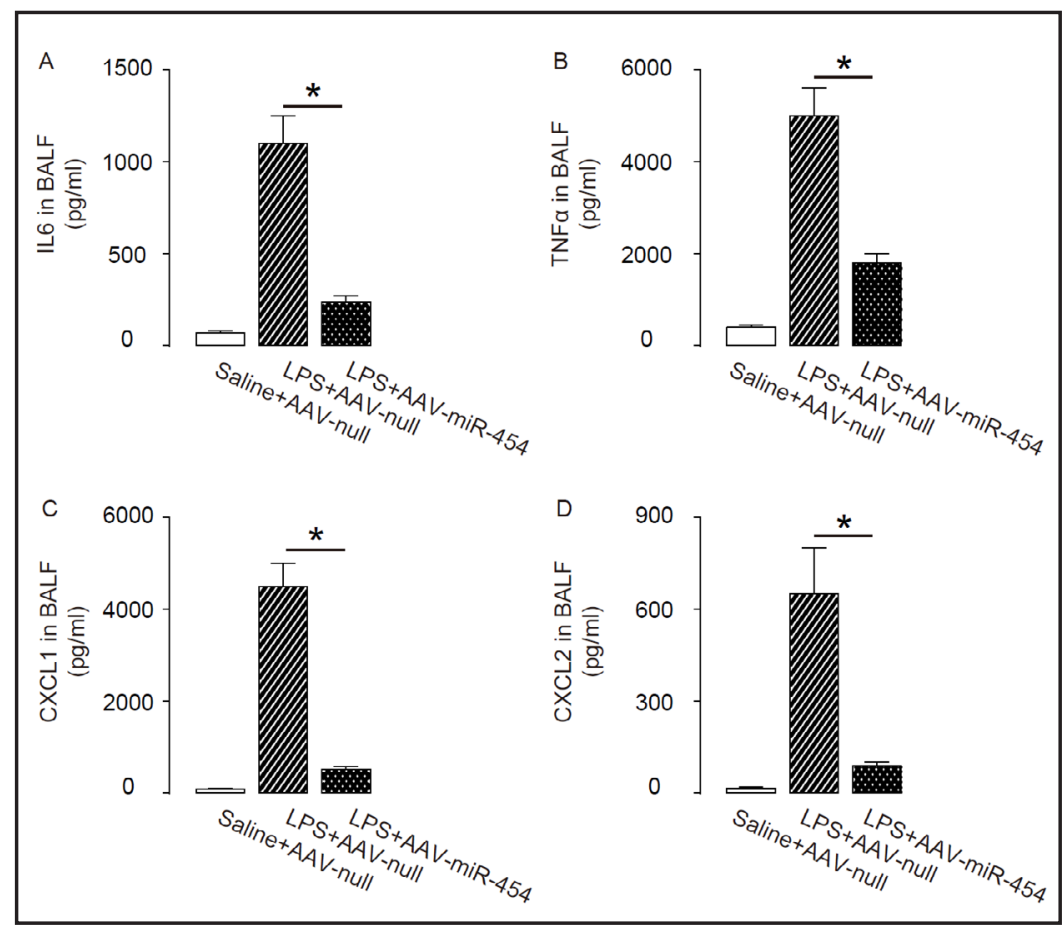


Fig. 4. Overexpression of miR454 in mouse lung decreases CXCL12 protein but not mRNA. The mouse lung was examined. (A) The levels of miR454 by RT-qPCR. (B) The CXCL12 transcripts by RT-qPCR. (C) The CXCL12 protein by ELISA. ${ }^{*} \mathrm{p}<0.05$, NS: non-significant, $\mathrm{N}=10$.

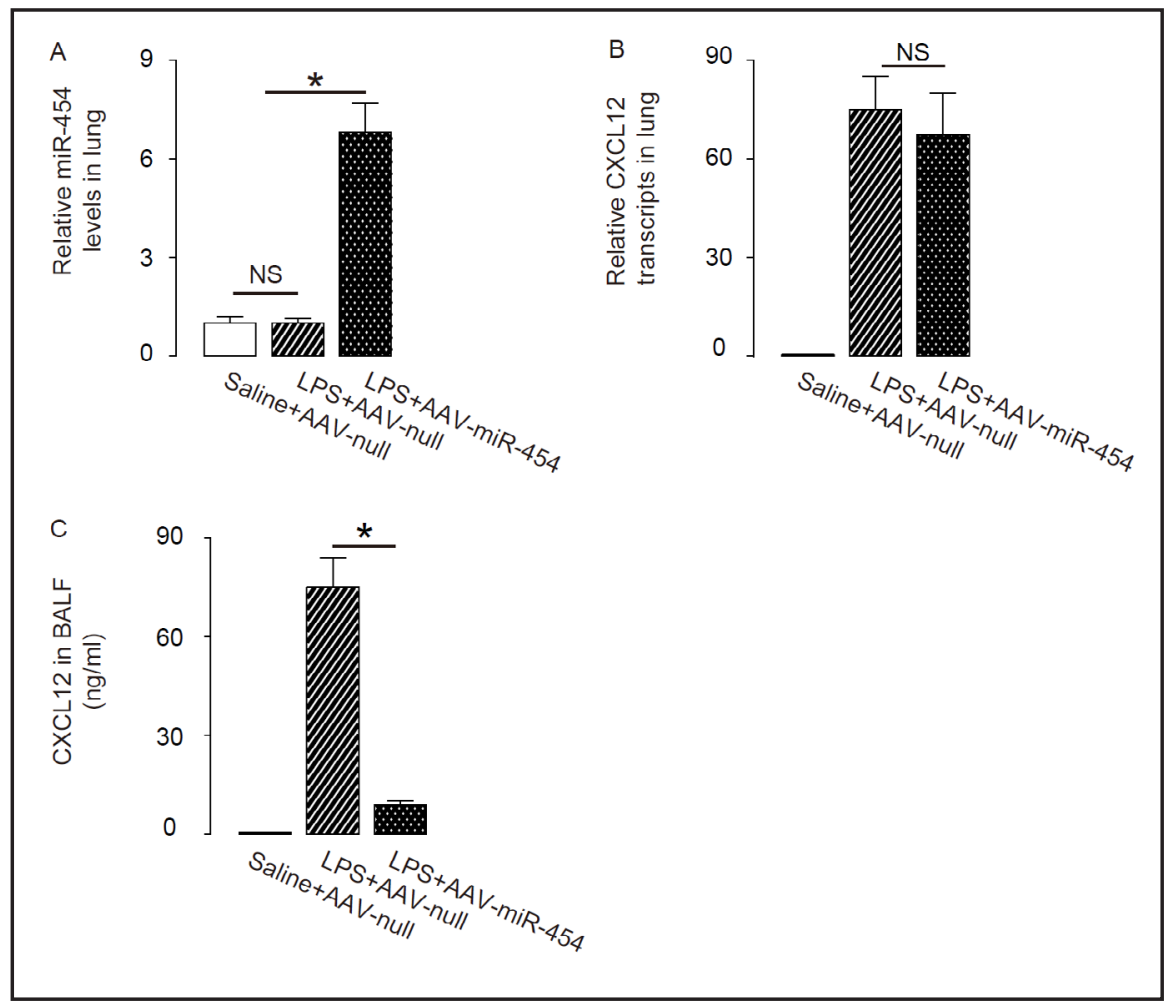

Fig. 5. Overexpression of miR454 in mouse lung reduces inflammation. (A-B) The mouse lung was digested and the CD45+ inflammatory cells in mouse lung were analyzed by flow cytometry, shown by representative flow charts (A), and by quantification (B). ${ }^{*} \mathrm{p}<0.05$, $\mathrm{N}=10$.
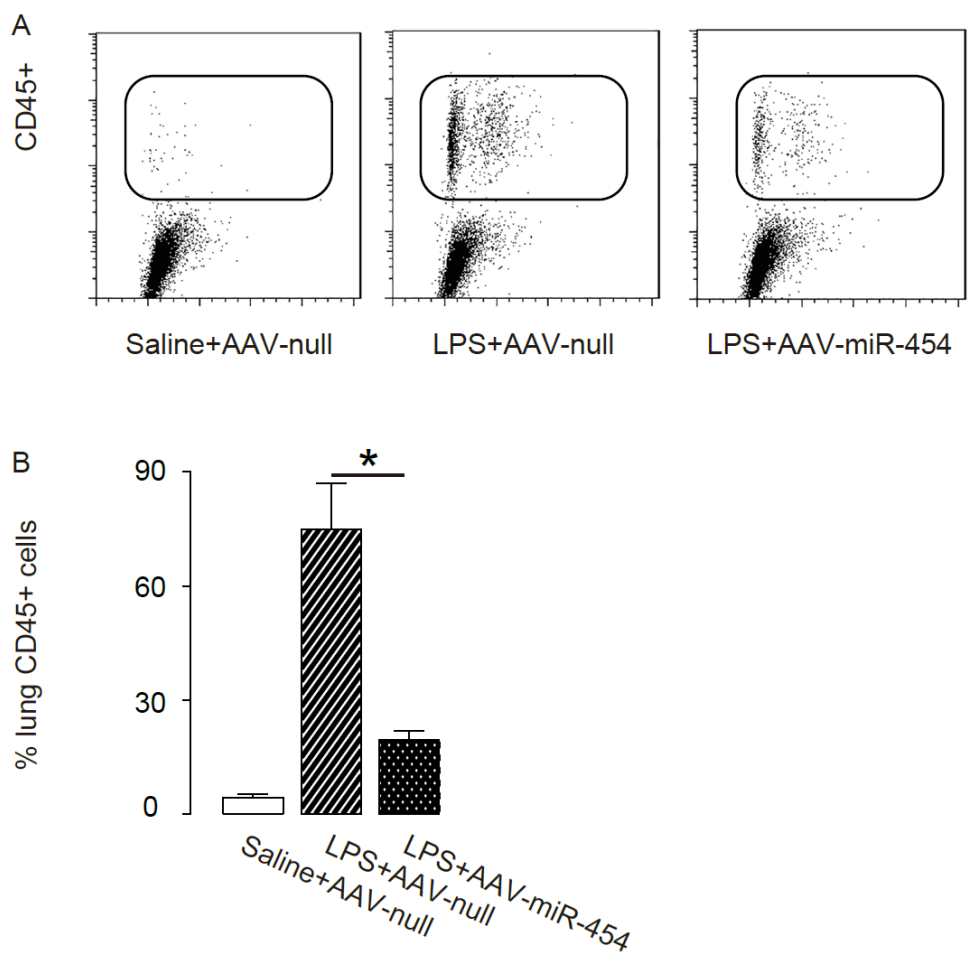

Overexpression of miR-454 in mouse lung reduces inflammation

Since CXCL12 is the chemo-attractive factor to recruit CXCR4+ inflammatory cells, we thus quantified the recruited inflammatory cells in mouse lung by flow cytometry based on CD45. We found that the CD45+ inflammatory cells in mouse lung were significantly reduced by miR-454 overexpression, shown by representative flow charts (Fig. 5A), and by quantification (Fig. 5B). Together, our data demonstrate that overexpression of miR- 


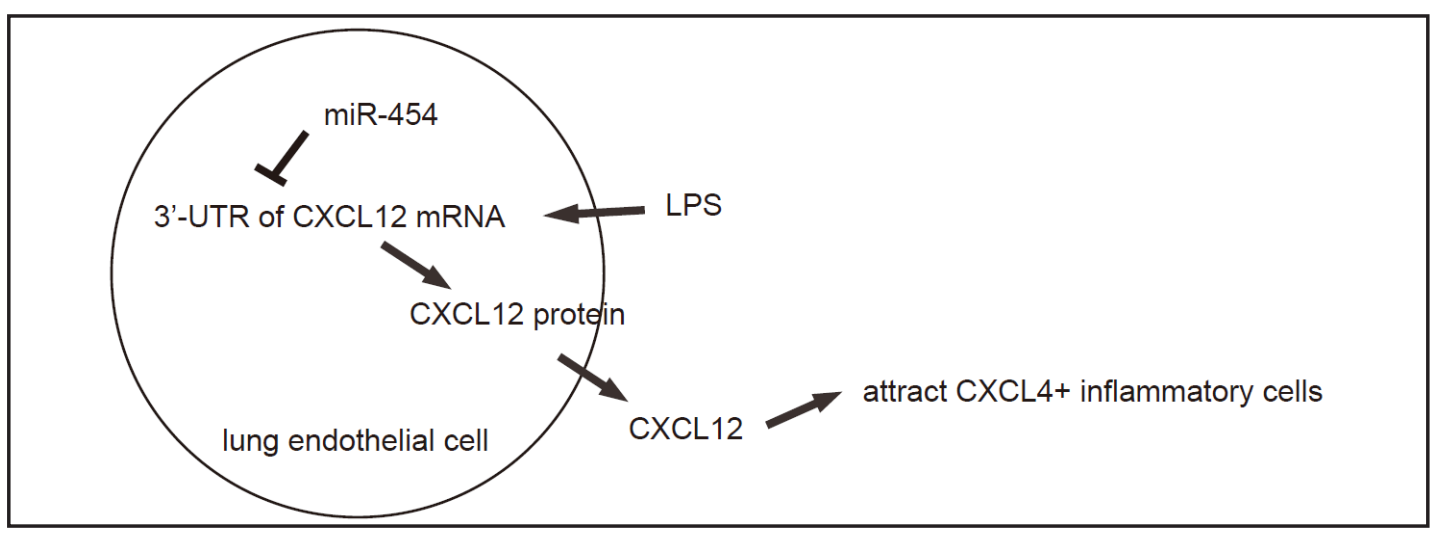

Fig. 6. A schematic model. Overexpression of miR-454 in mouse lung significantly reduces the LPS-induced increases in permeability index and production of inflammatory cytokines CXCL1, CXCL2, IL6 and TNF $\alpha$, possibly through suppression of CXCL12/CXCR4-mediated recruitment of inflammatory cells.

454 in mouse lung significantly reduces the LPS-induced increases in permeability index and production of inflammatory cytokines CXCL1, CXCL2, IL6 and TNF $\alpha$, possibly through suppression of CXCL12/CXCR4-mediated recruitment of inflammatory cells (Fig. 6).

\section{Discussion}

It is noteworthy that ALI-induced ARDS is one of the leading causes for the death of patients in intense care unit. The current ALI therapy is primarily made up of supportive treatments like ventilation, which have limited effects on severe cases [1-4]. Hence, innovative therapy is highly needed to improve the current ALI/ARDS treatments, and this therapeutic innovation requires identification of new targets, e.g. miRNAs.

The severe and protracted inflammation that characterizes ALI is driven by the ongoing recruitment of inflammatory cells to the lung. Although much of the cytokine signaling responsible for the initial phase of ALI has been elaborated, relatively few approaches have been figured out to target inflammation suppression. Given its previously described chemoattractant effects on inflammatory cells, we investigated whether CXCL12 might be a good target and might be effectively regulated by miRNAs.

In vitro, we performed bioinformatics analyses to identify the miRNAs that target the most CXCL12, and used luciferase reporter assay to confirm that the binding was functional. Interestingly, we only found one miRNA that binds to CXCL12, and this miRNA is miR-454. Moreover, we found that miR-454 binding sites on the 3'-UTR of CXCL12 mRNA ranged from $1466^{\text {th }}$ to $1472^{\text {nd }}$ base site, and this binding is functional. Thus, miR-454 is a unique CXCL12mRNA-targeting regulatory miRNA, which highlights its importance in CXCL12-related therapy.

We then used an AAV vector system to delivery miR-454 in mouse lung through circulation. This approach took advantages of that mouse lung is the predominant site for retention of systemic delivered virus, and that AAV induces less inflammation by themselves, compared to adenovirus $[21,22]$. Overexpression of miR-454 in lung was found to alleviate LPS-induced ALI by permeability index, by lung histology, and by the levels of inflammatory cytokines in the BALF. The effects of miR-454 on CXCL12 in vivo were confirmed by the fact that overexpression of miR-454 in mouse lung decreased CXCL12 protein but not mRNA. Finally, overexpression of miR-454 in mouse lung significantly reduced the LPS-induced increases in permeability index and production of inflammatory cytokines CXCL1, CXCL2, IL6 and TNF $\alpha$, possibly through suppression of CXCL12/CXCR4-mediated recruitment of inflammatory cells, as shown in Fig. 6. 


\section{Cellular Physiology Cell Physiol Biochem 2016;38:65-74 \begin{tabular}{l|l|l|}
\hline DOI: 10.1159/000438609 & $\begin{array}{l}\text { C) 2016 The Author(s). Published by S. Karger AG, Basel } \\
\text { www.karger.com/cpb }\end{array}$ \\
\hline
\end{tabular} \\ Tao/Yuan/Liao: Alleviation of LPS-Induced ALI by MiR-454}

Further experiments may be applied to evaluate the long-term effects of miR-454 overexpression in mouse lung after LPS treatment, specifically the long function should be examined. Moreover, it may be interesting to assess recovery of lung function, e.g. the changes in airway hyper responsiveness, after a low dose of LPS treatment of the mice. These approaches may greatly improve our understandings of the molecular basis of function protection of lung after infection-associated insults, and may shed light on development of novel treatments for ALI.

\section{Disclosure Statement}

The authors have declared that no conflict of interest exists.

\section{References}

1 Ashbaugh DG, Bigelow DB, Petty TL, Levine BE: Acute respiratory distress in adults. Lancet 1967;2:319323.

2 Herold S, Gabrielli NM, Vadasz I: Novel concepts of acute lung injury and alveolar-capillary barrier dysfunction. Am J Physiol Lung Cell Mol Physiol 2013;305:L665-681.

3 Dengler V, Downey GP, Tuder RM, Eltzschig HK, Schmidt EP: Neutrophil intercellular communication in acute lung injury. Emerging roles of microparticles and gap junctions. Am J Respir Cell Mol Biol 2013;49:15.

4 Vlaar AP, Juffermans NP: Transfusion-related acute lung injury: A clinical review. Lancet 2013;382:984994.

5 Deng X, Jin K, Li Y, Gu W, Liu M, Zhou L: Platelet-derived growth factor and transforming growth factor beta1 regulate ards-associated lung fibrosis through distinct signaling pathways. Cell Physiol Biochem 2015;36:937-946.

6 Ji Y, Gao F, Sun B, Hao J, Liu Z: Angiotensin-converting enzyme 2 inhibits apoptosis of pulmonary endothelial cells during acute lung injury through suppressing smad2 phosphorylation. Cell Physiol Biochem 2015;35:2203-2212.

7 Liu S, Tang J, Huang L, Xu Q Ling X, Liu J: Cordyceps militaris alleviates severity of murine acute lung injury through mirnas-mediated cxcr2 inhibition. Cell Physiol Biochem 2015;36:2003-2011.

8 Landgraf MA, Silva RC, Correa-Costa M, Hiyane MI, Carvalho MH, Landgraf RG, Camara NO: Leptin downregulates lps-induced lung injury: Role of corticosterone and insulin. Cell Physiol Biochem 2014;33:835-846.

9 Thambiayya K, Wasserloos K, Kagan VE, Stoyanovsky D, Pitt BR: A critical role for increased labile zinc in reducing sensitivity of cultured sheep pulmonary artery endothelial cells to lps-induced apoptosis. Am J Physiol Lung Cell Mol Physiol 2012;302:L1287-1295.

10 Martins JO, Ferracini M, Ravanelli N, Landgraf RG, Jancar S: Insulin suppresses lps-induced inos and cox-2 expression and nf-kappab activation in alveolar macrophages. Cell Physiol Biochem 2008;22:279-286.

11 Martins JO, Ferracini M, Ravanelli N, Landgraf RG, Jancar S: Insulin inhibits lps-induced signaling pathways in alveolar macrophages. Cell Physiol Biochem 2008;21:297-304.

12 Tettamanti G, Malagoli D, Benelli R, Albini A, Grimaldi A, Perletti G, Noonan DM, de Eguileor M, Ottaviani E: Growth factors and chemokines: A comparative functional approach between invertebrates and vertebrates. Curr Med Chem 2006;13:2737-2750.

13 Ruiz de Almodovar C, Luttun A, Carmeliet P: An sdf-1 trap for myeloid cells stimulates angiogenesis. Cell 2006;124:18-21.

14 Grunewald M, Avraham I, Dor Y, Bachar-Lustig E, Itin A, Jung S, Chimenti S, Landsman L, Abramovitch R, Keshet E: Vegf-induced adult neovascularization: Recruitment, retention, and role of accessory cells. Cell 2006;124:175-189.

15 Cao X, Han ZB, Zhao H, Liu Q: Transplantation of mesenchymal stem cells recruits trophic macrophages to induce pancreatic beta cell regeneration in diabetic mice. Int J Biochem Cell Biol 2014;53:372-379.

16 Di Leva G, Croce CM: Mirna profiling of cancer. Curr Opin Genet Dev 2013;23:3-11. 


\section{Cellular Physiology Cell Physiol Biochem 2016;38:65-74

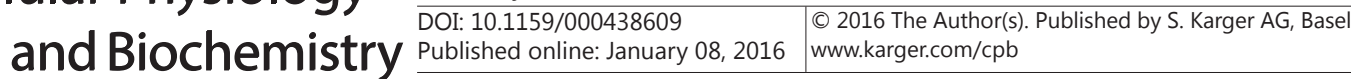 \\ Tao/Yuan/Liao: Alleviation of LPS-Induced ALI by MiR-454}

17 Pereira DM, Rodrigues PM, Borralho PM, Rodrigues CM: Delivering the promise of mirna cancer therapeutics. Drug Discov Today 2013;18:282-289.

18 Zhu D, He X, Duan Y, Chen J, Wang J, Sun X, Qian H, Feng J, Sun W, Xu F, Zhang L: Expression of microrna-454 in tgf-beta1-stimulated hepatic stellate cells and in mouse livers infected with schistosoma japonicum. Parasit Vectors 2014;7:148.

19 Ades EW, Candal FJ, Swerlick RA, George VG, Summers S, Bosse DC, Lawley TJ: Hmec-1: Establishment of an immortalized human microvascular endothelial cell line. J Invest Dermatol 1992;99:683-690.

20 Coronnello C, Benos PV: Comir: Combinatorial microrna target prediction tool. Nucleic Acids Res 2013;41:W159-164.

21 Xiao X, Guo P, Prasadan K, Shiota C, Peirish L, Fischbach S, Song Z, Gaffar I, Wiersch J, El-Gohary Y, Husain SZ, Gittes GK: Pancreatic cell tracing, lineage tagging and targeted genetic manipulations in multiple cell types using pancreatic ductal infusion of adeno-associated viral vectors and/or cell-tagging dyes. Nat Protoc 2014;9:2719-2724.

22 Khan IF, Hirata RK, Russell DW: Aav-mediated gene targeting methods for human cells. Nat Protoc 2011;6:482-501. 